\title{
'Classical' convergence theorems for generalized continued fractions
}

\author{
Marcel G. de Bruin
}

Received: 11 May 2007 / Accepted: 11 June 2007 /

Published online: 25 July 2007

(C) Springer Science + Business Media B.V. 2007

\begin{abstract}
In this paper the classical convergence theorems by ŚleszyńskiPringsheim, Worpitzky and Van Vleck for ordinary continued fractions will be generalized to continued fractions generalizations (along the lines of the Jacobi-Perron algorithm) with four-term recurrence relations.
\end{abstract}

Keywords Generalized continued fractions • Jacobi-Perron algorithm • Recurrence relations $\cdot$ Linear fractional transformations - Convergence

Mathematics Subject Classification (2000) MSC 40A15

\section{Introduction}

This paper is part of a project to extend some 'classical' convergence theorems for continued fractions, due to Śleszyński-Pringsheim, Worpitzky and Van Vleck, to the case of so-called $n$-fractions.

In order to understand the difficulties arising in the extension of the methods of proof, we consider in this paper the case $n=2$ : so-called 2-fractions. The paper is organized as indicated below.

In Section 2 the definition of these 2-fractions will be given, introducing the different types of approach: the Jacobi-Perron algorithm angle, a 4-term recurrence relation for the sequence of denominators and the two sequences of numerators, and the method of linear fractional transformations.

After this, extensions of three classical results will be given in Section 3, followed by the proofs in Section 4. After some concluding remarks in Section 5 a short list of references is given.

M. G. de Bruin $(\bowtie)$

Delft Institute for Applied Mathematics, P.O. Box 5031, 2600 GA Delft, The Netherlands e-mail: m.g.debruin@tudelft.nl 
Before starting with the theoretical framework for 2-fractions, the classical results to be studied will be formulated here in the forms as they appear in [9]:

Theorem 1 (Śleszyński-Pringsheim) The continued fraction $\mathbf{K}\left(a_{n} / b_{n}\right)$ converges if for all $n \geq 1$

$$
\left|b_{n}\right| \geq\left|a_{n}\right|+1 .
$$

Under the same conditions

$$
\left|f_{n}\right|<1
$$

holds for all approximants $f_{n}$, and

$$
|f| \leq 1
$$

for the value of the continued fraction.

Theorem 2 (Worpitzky) Let for all $n \geq 1$

$$
\left|a_{n}\right| \leq \frac{1}{4} .
$$

Then $\mathbf{K}\left(a_{n} / 1\right)$ converges. All approximants are in the disk

$$
|w|<\frac{1}{2},
$$

and the value $f$ is in the disk $|w| \leq \frac{1}{2}$.

Theorem 3 (Van Vleck) Let $0<\varepsilon<\pi / 2$, and let $b_{n}$ satisfy

$$
-\frac{\pi}{2}+\varepsilon<\arg b_{n}<\frac{\pi}{2}-\varepsilon
$$

for all $n$. Then all aproximants of $\mathbf{K}\left(1 / b_{n}\right)$ are finite and in the angular domain

$$
-\frac{\pi}{2}+\varepsilon<\arg f_{n}<\frac{\pi}{2}-\varepsilon .
$$

Furthermore, the sequences $\left\{f_{2 m}\right\}$ and $\left\{f_{2 m+1}\right\}$ converge to finite values.

If (and only if), in addition

$$
\sum_{n=1}^{\infty}\left|b_{n}\right|=\infty
$$

then $\mathbf{K}\left(1 / b_{n}\right)$ converges.

\section{Definition of a 2-fraction}

Let $\left(a_{i, k}\right), i=1,2$, and $\left(b_{k}\right)$ be given sequences of complex numbers satisfying

$$
a_{1, k} \neq 0, b_{k} \neq 0, \quad k \geq 1 .
$$


The symbol

$$
\underset{k=1}{\infty}\left[\begin{array}{c}
a_{1, k} \\
a_{2, k} \\
b_{k}
\end{array}\right]
$$

is used to denote a so-called 2-fraction, given by its sequences of pairs of approximants of 'rational numbers' with a common denominator

$$
f_{i, n}=\frac{A_{i, n}}{b_{n}}, i=1,2 ; n \geq 0 .
$$

The calculation of these approximants can be done in different manners that will be discussed below. Because we are dealing with approximation, the following will not come as a surprise:

Definition 1 A 2-fraction (10) for which both limits

$$
\lim _{n \rightarrow \infty} f_{i, n}=f_{i}(i=1,2),
$$

exist and are finite, is called convergent. Otherwise it is called divergent.

\subsection{Jacobi-Perron algorithm approach}

The 'construction' of these approximant pairs, apart from $f_{i, 0}=0(i=1,2)$, can be seen from

$$
\begin{aligned}
& f_{1,1}=\frac{a_{1,1}}{b_{1}}, \quad f_{1,2}=\frac{a_{1,1}}{b_{1}+\frac{a_{2,2}}{b_{2}},} \quad f_{1,3}=\frac{a_{1,1}}{b_{1}+\frac{a_{2,2}+\frac{a_{1,3}}{b_{3}}}{b_{2}+\frac{a_{2,3}}{b_{3}}}, \ldots} \\
& f_{2,1}=\frac{a_{2,1}}{b_{1}}, \quad f_{2,2}=\frac{a_{2,1}+\frac{a_{1,2}}{b_{2}}}{b_{1}+\frac{a_{2,2}}{b_{2}}}, \quad f_{2,3}=\frac{a_{1,2}}{b_{2}+\frac{a_{2,3}}{b_{3}}}, \ldots \\
& b_{1}+\frac{a_{2,2}+\frac{a_{1,3}}{b_{3}}}{b_{2}+\frac{a_{2,3}}{b_{3}}}
\end{aligned}
$$

In each step the replacement of the final entries in the form for $f_{i, k}$ to find the form for $f_{i, k+1}$ is given by

$$
a_{1, k} \mapsto a_{1, k}, a_{2, k} \mapsto a_{2, k}+\frac{a_{1, k+1}}{b_{k+1}}, b_{k} \mapsto b_{k}+\frac{a_{2, k+1}}{b_{k+1}}
$$


Remark This construction, already present in the original introduction of the Jacobi-Perron algorithm in [1], has been used before in [3]. There is a fundamental difference with the approach used in branched continued fractions, $[2,5,8,10]$.

\subsection{Recurrence relation approach}

The numerator- and denominator-sequences all satisfy the same recurrence relation

$$
X_{k}=b_{k} X_{k-1}+a_{2, k} X_{k-2}+a_{1, k} X_{k-3}, k \geq 1,
$$

with initial values

$$
\begin{aligned}
A_{1,-2} & =1, & A_{1,-1} & =0, & A_{1,0} & =0, \\
A_{2,-2} & =0, & A_{2,-1} & =1, & A_{2,0} & =0, \\
B_{-2} & =0, & B_{-1} & =0, & B_{0} & =1 .
\end{aligned}
$$

The proof of (15) follows easily from (16), (13) and the 'replacement rules' in (14).

Remark In [3] these formulae and the 'iterated matrix approach', already present in [1], have been used to study generalized $C$-fractions: a type of continued fractions that appear in a multi-dimensional Padé table.

\subsection{Linear fractional transformation approach}

Using the coefficients indicated in the array (10) introduce the LFT-s, cf. [4]:

$$
s_{k}^{(1)}\left(y_{1}, y_{2}\right)=\frac{a_{1, k}}{b_{k}+y_{2}}, s_{k}^{(2)}\left(y_{1}, y_{2}\right)=\frac{a_{2, k}+y_{1}}{b_{k}+y_{2}} \quad(k \geq 1),
$$

and for $i=1,2, k \geq 1$ the iterated transforms

$$
S_{1}^{(i)}\left(y_{1}, y_{2}\right)=s_{1}^{(i)}\left(y_{1}, y_{2}\right) ; S_{k}^{(i)}\left(y_{1}, y_{2}\right)=S_{k-1}^{(i)}\left(s_{k}^{(1)}\left(y_{1}, y_{2}\right), s_{k}^{(2)}\left(y_{1}, y_{2}\right)\right)(k \geq 2) \text {. }
$$

Using induction ((16) for $S_{1}^{(i)}$ and (15) for the induction step) it is easy to prove

Theorem 4 For $i=1,2, k \geq 1$ :

$$
S_{k}^{(i)}\left(y_{1}, y_{2}\right)=\frac{A_{i, k}+y_{2} A_{i, k-1}+y_{1} A_{i, k-2}}{B_{k}+y_{2} B_{k-1}+y_{1} B_{k-2}} .
$$

Remark Thus for $i=1,2$ :

$$
S_{k}^{(i)}(0,0)=\frac{A_{i, k}}{B_{k}}, \quad S_{k}^{(i)}(0, \infty)=\frac{A_{i, k-1}}{B_{k-1}}(k \geq 1), \quad S_{k}^{(i)}(\infty, 0)=\frac{A_{i, k-2}}{B_{k-2}}(k \geq 2) .
$$




\section{Convergence results}

As a generalization of the famous Śleszyński-Pringsheim theorem (Theorem 1) for ordinary continued fractions (see [9]) we have

Theorem 5 Given real numbers $\alpha_{1}, \alpha_{2}>0$ with

$$
\frac{1}{2}\left(\sqrt{\alpha_{2}^{2}+4 \alpha_{1}}+\alpha_{2}\right)<\frac{\alpha_{1}}{\alpha_{2}} \Leftrightarrow 2 \alpha_{2}^{2}<\alpha_{1},
$$

and let the coefficients of the 2-fraction (10) satisfy

$$
\left|b_{k}\right| \geq \frac{1}{\alpha_{1}}\left|a_{1, k}\right|+\alpha_{2},\left|b_{k}\right| \geq \frac{1}{\alpha_{2}}\left(\left|a_{2, k}\right|+\alpha_{1}\right)+\alpha_{2}, k \geq 1 .
$$

Then the approximant pairs satisfy

$$
\left|f_{1, n}\right|<\alpha_{1},\left|f_{2, n}\right|<\alpha_{2}, n \geq 1 .
$$

Moreover, the 2-fraction is convergent and the limits satisfy

$$
\left|f_{1}\right| \leq \alpha_{1},\left|f_{2}\right| \leq \alpha_{2} .
$$

Remark The form of the conditions in (22) arises naturally from the connection between the approximants in (13) and the linear fractional transformations (17).

As a simple corollary of this, we find a generalization of a result due to Worpitzky (Theorem 2; see also [9]).

Theorem 6 Let the positive real numbers $\alpha_{1}, \alpha_{2}$ and $\rho$ satisfy

$$
\alpha_{1}>2 \alpha_{2}^{2}, \rho>\alpha_{2}+\frac{\alpha_{1}}{\alpha_{2}} .
$$

Then a 2-fraction of the form

$$
\underset{k=1}{\infty}\left[\begin{array}{c}
a_{1, k} \\
a_{2, k} \\
1
\end{array}\right]
$$

with

$$
\left|a_{1, n}\right| \leq \frac{\left(\rho-\alpha_{2}\right) \alpha_{1}}{\rho^{3}},\left|a_{2, n}\right| \leq \frac{\alpha_{2} \rho-\alpha_{1}-\alpha_{2}^{2}}{\rho^{2}}(n \geq 1),
$$

is convergent and its approximants and limits satisfy

$$
\begin{gathered}
f_{1, n}=\frac{A_{1, n}}{B_{n}} \in \mathbf{D}\left(0 ; \frac{\alpha_{1}}{\rho^{2}}\right), f_{1} \in \overline{\mathbf{D}\left(0 ; \frac{\alpha_{1}}{\rho^{2}}\right)}, \\
f_{2, n}=\frac{A_{2, n}}{B_{n}} \in \mathbf{D}\left(0 ; \frac{\alpha_{2}}{\rho}\right), f_{2} \in \overline{\mathbf{D}\left(0 ; \frac{\alpha_{2}}{\rho}\right)} .
\end{gathered}
$$


N.B. $\mathbf{D}(a, R)=\{z \in \mathbb{C}|| z-a \mid<R\}$ and the overline indicates the closed disk.

Remark A different generalization of Theorem 5 has been given in [7]. The conditions (22) above are replaced by

$$
\left|b_{k}\right| \geq 1+\left|a_{1, k}\right|+\left|a_{2, k}\right|(k \geq 1) .
$$

Letting $\alpha_{1}, \alpha_{2}$ range over $(0, \infty)$ in Theorem 5 , it can be seen that (29) is less restrictive than (22) for certain sets of values of $a_{i, k}$ only (example: for $\left|a_{1, k}\right| \geq$ $2+\delta>2, a_{2, k}$ arbitrary, the conditions (22) with $\alpha_{1}=2+\delta / 2, \alpha_{2}=1$ are less restrictive than (29)).

Finally we have a partial generalization of a theorem due to Van Vleck (Theorem 3; see also [9]):

Theorem 7 Let $A \doteq 0.4516059630$ be the unique positive root of $2 A^{3}+4 A^{2}-$ $1=0$ and let the 2-fraction of the form

$$
\underset{k=1}{\infty}\left[\begin{array}{c}
1 \\
1 \\
b_{k}
\end{array}\right]
$$

satisfy

$$
\boldsymbol{\operatorname { R e }} b_{k} \geq 2 \sqrt{\frac{A}{2}} \doteq 0.9503746240 .
$$

Let the half-plane $\mathbf{H}$ and parabolic region $\mathbf{P}$ be given by

$$
\mathbf{H}=\{z \in \mathbb{C} \mid \operatorname{Re} z>-\sqrt{A / 2}\}, \mathbf{P}=\{z \in \mathbb{C}|\operatorname{Re}(1+z)-| 1+z \mid>-A\} .
$$

Then the approximants $f_{i, n}$ are all finite and satisfy

$$
f_{1, n} \in \mathbf{H} \cap \mathbf{P}, f_{2, n} \in \mathbf{H},
$$

\section{Proofs}

The following quantities play (as was to be expected) an important role:

$$
\Delta_{i, k}=A_{i, k} B_{k-1}-A_{i, k-1} B_{k}(i=1,2 ; k \geq-1) .
$$

They satisfy

Lemma 1 The $\Delta_{i, k}$ satisfy the recurrence relation

$$
X_{k}=-a_{2, k} X_{k-1}-a_{1, k} b_{k-1} X_{k-2}+a_{1, k} a_{1, k-1} X_{k-3}(k \geq 2),
$$

with initial values

$$
\begin{aligned}
& \Delta_{1,-1}=0, \Delta_{1,0}=0, \Delta_{1,1}=a_{1,1} \\
& \Delta_{2,-1}=0, \Delta_{2,0}=-1, \Delta_{2,1}=a_{2,1}
\end{aligned}
$$


Proof The initial values (36) follow from the values (16) immediately and equation (35) follows from application of the recurrence relation (15) twice in the following manner.

Combine

$$
\begin{aligned}
X_{k}= & A_{i, k} B_{k-1}-A_{i, k-1} B_{k} \\
= & \left(b_{k} A_{i, k-1}+a_{2, k} A_{i, k-2}+a_{1, k} A_{i, k-3}\right) B_{k} \\
& -A_{i, k-1}\left(b_{k} B_{k-1}+a_{2, k} B_{k-2}+a_{1, k} B_{k-3}\right) \\
= & -a_{2, k}\left(A_{i, k-1} B_{k-2}-A_{i, k-2} B_{k-1}\right) \\
& -a_{1, k}\left(A_{i, k-1} B_{k-3}-A_{i, k-3} B_{k-1}\right)(k \geq 1),
\end{aligned}
$$

for a fixed $k \geq 1$ with

$$
\begin{aligned}
A_{i, k} & B_{k-2}-A_{i, k-2} B_{k}= \\
= & \left(b_{k} A_{i, k-1}+a_{2, k} A_{i, k-2}+a_{1, k} A_{i, k-3}\right) B_{k-2} \\
& -A_{i, k-2}\left(b_{k} B_{k-1}+a_{2, k} B_{k-2}+a_{1, k} B_{k-3}\right) \\
= & b_{k}\left(A_{i, k-1} B_{k-2}-A_{i, k-2} B_{k-1}\right) \\
& -a_{1, k}\left(A_{i, k-2} B_{k-3}-A_{i, k-3} B_{k-2}\right)(k \geq 1),
\end{aligned}
$$

for $k-1 \geq 1$, i.e. $k \geq 2$ and we find (35).

Remark Equation (35) is called the adjoint of (15); cf. [6].

Now introduce the quantities $\delta_{i, k}$ by

$$
\begin{aligned}
& \delta_{1,-1}=0, \delta_{1,0}=0, \delta_{1,1}=\alpha_{1}\left(\left|b_{1}\right|-\alpha_{2}\right) \\
& \delta_{2,-1}=0, \delta_{2,0}=1, \delta_{2,1}=\alpha_{2}\left(\left|b_{1}\right|-\alpha_{2}\right)-\alpha_{1}
\end{aligned}
$$

and

$$
\begin{aligned}
\delta_{i, k}= & \left.\left\{\alpha_{2}\left(\left|b_{k}\right|-\alpha_{2}\right)-\alpha_{1}\right)\right\} \delta_{i, k-1}+\left\{\alpha_{1}\left(\left|b_{k}\right|-\alpha_{2}\right)\right\}\left|b_{k-1}\right| \delta_{i, k-2} \\
& +\alpha_{1}^{2}\left(\left|b_{k}\right|-\alpha_{2}\right)\left(\left|b_{k-1}\right|-\alpha_{2}\right) \delta_{i, k-3}(k \geq 2),
\end{aligned}
$$

then

Lemma 2 Let the sequence $\left(c_{j}\right)_{j}$ be given by

$$
c_{-1}=0, c_{0}=1 ; c_{j}=\alpha_{2} c_{j-1}+\alpha_{1} c_{j-2}(j \geq 1),
$$

then the quantities $\delta_{i, n}$ satisfy

$$
0<\delta_{1, n}=\sum_{j=0}^{n}(-1)^{j} \alpha_{1}^{j+1} c_{n-1-j} \prod_{k=1}^{n-j}\left(\left|b_{k}\right|-\alpha_{2}\right) \leq \alpha_{1} c_{n-1} \prod_{k=1}^{n}\left(\left|b_{k}\right|-\alpha_{2}\right)(n \geq 0),
$$


and

$$
0<\delta_{2, n}=\sum_{j=0}^{n}(-1)^{j} \alpha_{1}^{j} c_{n-j} \prod_{k=1}^{n-j}\left(\left|b_{k}\right|-\alpha_{2}\right) \leq c_{n} \prod_{k=1}^{n}\left(\left|b_{k}\right|-\alpha_{2}\right)(n \geq 0) .
$$

Moreover

$$
\left|\Delta_{i, k}\right| \leq \delta_{i, k}(i=1,2 ; k \geq-1)
$$

Proof Put

$$
d_{k}=\left|b_{k}\right|-\alpha_{2}(k \geq 1)
$$

then (40) can be written as

$$
\delta_{i, k}=\left(\alpha_{2} d_{k}-\alpha_{1}\right) \delta_{i, k-1}+\alpha_{1} d_{k}\left(d_{k-1}+\alpha_{2}\right) \delta_{i, k-2}+\alpha_{1}^{2} d_{k} d_{k-1} \delta_{i, k-3},
$$

or

$$
\delta_{i, k}+\alpha_{1} \delta_{i, k-1}=\alpha_{2} d_{k}\left(\delta_{i, k-1}+\alpha_{1} \delta_{i, k-2}\right)+\alpha_{1} d_{k} d_{k-1}\left(\delta_{i, k-2}+\alpha_{1} \delta_{i, k-3}\right) .
$$

Thus

$$
u_{i, k}=\frac{\delta_{i, k}+\alpha_{1} \delta_{i, k-1}}{\prod_{i=1}^{k} d_{k}}
$$

is a solution of the recurrence relation

$$
u_{k}=\alpha_{2} u_{k-1}+\alpha_{1} u_{k-2}(k \geq 2),
$$

with initial values

$$
u_{1,0}=0, u_{1,1}=\alpha_{1} ; u_{2,0}=1, u_{2,1}=\alpha_{2} .
$$

This immediately implies from (41)

$$
u_{1, n}=\alpha_{1} c_{n-1}, u_{2, n}=c_{n},
$$

and then the formulae

$$
\delta_{1, n}+\alpha_{1} \delta_{1, n-1}=\prod_{k=1}^{n}\left(\left|b_{k}\right|-\alpha_{2}\right) \cdot \alpha_{1} c_{n-1}, \delta_{2, n}+\alpha_{1} \delta_{2, n-1}=\prod_{k=1}^{n}\left(\left|b_{k}\right|-\alpha_{2}\right) \cdot c_{n},
$$

lead to $(42,43)$.

Proof of Theorem 5 The bounds (22) on the coefficients of the 2-fraction can be written as

$$
\left|a_{1, k}\right| \leq \alpha_{1}\left(\left|b_{k}\right|-\alpha_{2}\right), \quad\left|a_{2, k}\right| \leq \alpha_{2}\left(\left|b_{k}\right|-\alpha_{2}\right)-\alpha_{1}(k \geq 1) .
$$

Thus

$$
\left|\frac{a_{1, k}}{b_{k}}\right| \leq \frac{\alpha_{1}\left|b_{k}\right|-\alpha_{2}}{\left|b_{k}\right|}<\alpha_{1}, \quad\left|\frac{a_{2, k}}{b_{k}}\right| \leq \frac{\alpha_{2}\left(\left|b_{k}\right|-\alpha_{2}\right)-\alpha_{1}}{\left|b_{k}\right|}<\alpha_{2}(k \geq 1) .
$$


The pairs of approximations from (13) then satisfy

$$
\left\{\begin{array}{c}
\left|f_{1,1}\right|<\alpha_{1}, \quad\left|f_{2,1}\right|<\alpha_{2}, \\
\left|f_{1,2}\right| \leq \frac{\left|a_{1,1}\right|}{\left|b_{1}\right|-\left|\frac{a_{2,2}}{b_{2}}\right|}<\frac{\left|a_{1,1}\right|}{\left|b_{1}\right|-\alpha_{2}} \leq \alpha_{1}, \\
\left|f_{2,2}\right| \leq \frac{\left|a_{2,1}\right|+\left|\frac{a_{1,2}}{b_{2}}\right|}{\left|b_{1}\right|-\left|\frac{a_{2,2}}{b_{2}}\right|}<\frac{\left|a_{2,1}\right|+\alpha_{1}}{\left|b_{2}\right|-\alpha_{2}} \leq \alpha_{2} .
\end{array}\right.
$$

Because of the construction of approximations, see (14), we find (23).

For convergence we apply the usual approach

$$
\lim _{n \rightarrow \infty} \frac{A_{i, n}}{B_{n}} \text { exists } \Leftrightarrow \sum_{n=1}^{\infty}\left(\frac{A_{i, n}}{B_{n}}-\frac{A_{i, n-1}}{B_{n-1}}\right) \text { is convergent }(i=1,2) .
$$

As

$$
\left|\frac{A_{i, n}}{B_{n}}-\frac{A_{i, n-1}}{B_{n-1}}\right|=\frac{\left|\Delta_{i, n}\right|}{\left|B_{n}\right|\left|B_{n-1}\right|},
$$

an upper bound for the numerator is needed (this will be derived using Lemma 2) and a lower bound for $\left|b_{n}\right|$.

First the numerator: it follows easily from (41) that

$$
\sum_{n=0}^{\infty} c_{n} t^{n}=\frac{1}{1-\alpha_{2} t-\alpha_{1} t^{2}} .
$$

Now the reciprocals of the zeros of the denominator

$$
\rho=\frac{1}{2}\left(\sqrt{\alpha_{2}^{2}+4 \alpha_{1}}+\alpha_{2}\right), \sigma=\frac{1}{2}\left(\sqrt{\alpha_{2}^{2}+4 \alpha_{1}}-\alpha_{2}\right)
$$

lead with partial fraction decomposition of (54) to

$$
\sum_{n=0}^{\infty} c_{n} t^{n}=\frac{1}{\sqrt{\alpha_{2}^{2}+4 \alpha_{1}}} \sum_{n=0}^{\infty}\left\{\rho^{n+1}+(-1)^{n} \sigma^{n+1}\right\},
$$

or

$$
c_{n}=\frac{1}{\sqrt{\alpha_{2}^{2}+4 \alpha_{1}}}\left\{\rho^{n+1}+(-1)^{n} \sigma^{n+1}\right\}, n \geq 0 .
$$

From (57) we find the bounds

$$
0<c_{n}<\frac{2 \rho^{n+1}}{\sqrt{\alpha_{2}^{2}+4 \alpha_{1}}}, n \geq 0 .
$$


Using $d_{n}$ from (45), the recurrence relation

$$
y_{k}-\alpha_{2} y_{k-1}-\alpha_{1} y_{k-2}=\prod_{i=1}^{k} d_{k} ; y_{-1}=0, y_{0}=1
$$

has solution

$$
y_{n}=\sum_{j=0}^{n} c_{j} \prod_{r=1}^{n-j} d_{r}
$$

Now (15) and (49) imply

$$
\left|B_{n}\right|-\alpha_{2}\left|B_{n-1}\right|-\alpha_{1}\left|B_{n-2}\right| \geq\left(\left|b_{n}\right|-\alpha_{2}\right)\left(\left|B_{n-1}\right|-\alpha_{2}\left|B_{n-2}\right|-\alpha_{1}\left|B_{n-3}\right|\right),
$$

leading to

$$
\left|B_{n}\right|-\alpha_{2}\left|B_{n-1}\right|-\alpha_{1}\left|B_{n-2}\right| \geq \prod_{k=1}^{n}\left(\left|b_{k}\right|-\alpha_{2}\right),
$$

from which induction leads to

$$
\left|B_{n}\right| \geq y_{n}=\sum_{j=0}^{n} c_{j} \prod_{r=0}^{n-j}\left(\left|b_{r}\right|-\alpha_{2}\right),
$$

where the coefficients $c_{j}$ are the quantities defined in Lemma 2.

Using $\left|b_{k}\right| \geq \alpha_{1} / \alpha_{2}$ (from (22)), (34), (36), (42), (43), (44), (58), (62) and

$$
\begin{aligned}
\left|\frac{A_{i, n}}{B_{n}}-\frac{A_{i, n-1}}{B_{n-1}}\right| & =\frac{\left|\Delta_{i, n}\right|}{\left|B_{n}\right|\left|B_{n-1}\right|} \leq \\
& \leq \frac{\max \left\{\alpha_{1}, \rho\right\} 2 \rho^{n+1} \prod_{k=1}^{n}\left(\left|b_{k}\right|-\alpha_{2}\right) / \sqrt{\alpha_{2}^{2}+4 \alpha_{1}}}{\prod_{k=1}^{n}\left(\left|b_{k}\right|-\alpha_{2}\right) \times \prod_{k=1}^{n-1}\left(\left|b_{k}\right|-\alpha_{2}\right)} \leq \\
& \leq \frac{2 \rho \alpha_{1}}{\alpha_{2} \sqrt{\alpha_{2}^{2}+4 \alpha_{1}}} \max \left\{\alpha_{1}, \rho\right\}\left(\frac{\rho \alpha_{2}}{\alpha_{1}}\right)^{n},
\end{aligned}
$$

and the theorem follows from the conditions (21).

Proof of Theorem 6 This follows in the same manner as for ordinary continued fractions from Theorem 5 and a multiplier method for generalized continued fractions from [3].

Denote the numerators and denominators of

$$
\underset{\mathbf{K}}{\infty}\left[\begin{array}{c}
a_{1, k} \\
a_{2, k} \\
1
\end{array}\right]
$$

by

$$
A_{1, n}, A_{2, n}, \quad B_{n}(n \geq 0) .
$$


Then the denominators of the 2-fraction

$$
\underset{k=1}{\mathbf{K}}\left[\begin{array}{c}
a_{1, k} \rho_{k} \rho_{k-1} \rho_{k-2} \\
a_{2, k} \rho_{k} \rho_{k-1} \\
\rho_{k}
\end{array}\right],
$$

where $\rho_{k}>0(k \geq-1)$, are given by

$$
\begin{aligned}
\hat{A}_{1, n} & =A_{1, n} \rho_{-1} \rho_{0} \rho_{1} \cdots \rho_{n}(n \geq 0), \\
\hat{A}_{2, n} & =A_{2, n} \rho_{0} \rho_{1} \cdots \rho_{n}(n \geq 0), \\
\hat{B}_{n} & =B_{n} \rho_{1} \cdots \rho_{n}(n \geq 0) .
\end{aligned}
$$

Thus

$$
\frac{\hat{A}_{1, n}}{\hat{B}_{n}}=\rho_{0} \rho_{-1} \frac{A_{1, n}}{B_{n}}, \frac{\hat{A}_{2, n}}{\hat{B}_{n}}=\rho_{0} \frac{A_{2, n}}{B_{n}} \quad(n \geq 0) .
$$

With (27) and $\rho_{n}=\rho, n \geq-1$, the coefficients of (66) satisfy

$$
\begin{aligned}
\frac{1}{\alpha_{1}}\left|a_{1, k} \rho_{k} \rho_{k-1} \rho_{k-2}\right|+\alpha_{2} & \leq \rho=\rho_{k}, \\
\frac{1}{\alpha_{2}}\left(\left|a_{2, k} \rho_{k} \rho_{k-1}\right|+\alpha_{1}\right)+\alpha_{2} & \leq \rho=\rho_{k},
\end{aligned}
$$

and Theorem 5 implies that the 2-fraction (64) converges with

$$
\frac{\hat{A}_{1, n}}{\hat{B}_{n}} \in \mathbf{D}\left(0 ; \alpha_{1}\right), \frac{\hat{A}_{2, n}}{\hat{B}_{n}} \in \mathbf{D}\left(0 ; \alpha_{2}\right) .
$$

Then (68) implies (28).

Proof of Theorem 7 We start with real numbers

$$
\delta>0, A>0,
$$

and define the half-planes and parabolic region

$$
\mathbf{H}_{ \pm \delta}=\{z \in \mathbb{C} \mid \operatorname{Re} z> \pm \delta\}, \mathbf{P}=\{z \in \mathbb{C}|\operatorname{Re}(1+z)-| 1+z \mid>-A\} .
$$

Assuming

$$
b_{k} \in \mathbf{H}_{\delta},
$$

i.e.

$$
x_{k}:=\operatorname{Re} b_{k}>\delta,
$$

the proof consists of showing

$$
\left(y_{1}, y_{2}\right) \in\left(\mathbf{H}_{-\delta} \cap \mathbf{P}\right) \times \mathbf{H}_{-\delta} \Rightarrow\left(s_{k}^{(1)}\left(y_{1}, y_{2}\right), s_{k}^{(2)}\left(y_{1}, y_{2}\right)\right) \in\left(\mathbf{H}_{-\delta} \cap \mathbf{P}\right) \times \mathbf{H}_{-\delta} .
$$


The conditions (31) and the form of the sets in (32) will become clear 'on the run'.

A. The image of $\mathbf{H}_{-\delta}$ under $s_{k}^{(1)}\left(y_{1}, y_{2}\right)=1 /\left(b_{k}+y_{2}\right)$.

From the theory of linear fractional transformations it is known that the image of the half plane $\operatorname{Re} y_{2}>-\delta$ is a circle with center $M_{k}$ and radius $R_{k}$ given by:

- $M_{k}$ is the image of the point $z_{k}$ that forms together with the pole $-b_{k}$ a pair of symmetric points with respect to the boundary of the half-plane,

- $\quad R_{k}$ is the distance from $M_{k}$ to the image of an arbitrary point on the boundary of the half-plane; for instance

$$
\operatorname{dist}\left(M_{k}, s_{k}^{(1)}\left(y_{1}, \infty\right)=\left|M_{k}\right| .\right.
$$

The distance from $-b_{k}$ to $\operatorname{Re} y_{2}=-\delta$ is $\operatorname{Re} b_{k}-\delta$, leading to $z_{k}=-b_{k}+$ $2\left(\operatorname{Re} b_{k}-\delta\right)=\bar{b}_{k}-2 \delta$, implying

$$
M_{k}=\frac{1}{b_{k}+\left(\overline{b_{k}}-2 \delta\right)}=\frac{1}{2\left(x_{k}-\delta\right)}, R_{k}=\frac{1}{2\left(x_{k}-\delta\right)} .
$$

To find that the image belongs to $\mathbf{H}_{-\delta}$ again, we use (71):

$$
\operatorname{Re} M_{k}-R_{k}=0>-\delta \text {. }
$$

B. The image of $\mathbf{H}_{-\delta} \cap \mathbf{P}$ under $s_{k}^{(1)}\left(y_{1}, y_{2}\right)=1 /\left(b_{k}+y_{2}\right)$.

As we just saw that $\mathbf{H}_{-\delta}$ is mapped into itself, it is sufficient to prove that the boundary of the image (a disk) derived under $\mathbf{A}$ already satisfies:

$$
\operatorname{Re}\left(1+\frac{1+e^{i \varphi}}{2\left(x_{k}-\delta\right)}\right)-\left|1+\frac{1+e^{i \varphi}}{2\left(x_{k}-\delta\right)}\right| \geq-A, \varphi \in[0,2 \pi),
$$

or, equivalently

$$
1+\frac{1+\cos \varphi}{2\left(x_{k}-\delta\right)}+A \geq \sqrt{\left(1+\frac{1+\cos \varphi}{2\left(x_{k}-\delta\right)}\right)^{2}+\left(\frac{\sin \varphi}{2\left(x_{k}-\delta\right)}\right)^{2}},
$$

leading to

$$
2 A\left(1+\frac{1+\cos \varphi}{2\left(x_{k}-\delta\right)}\right)+A^{2} \geq \frac{\sin ^{2} \varphi}{4\left(x_{k}-\delta\right)^{2}} .
$$

Taking the minimal value (as a function of $\varphi$ ) on the left hand side and the maximum on the right hand side, we find the condition on $x_{k}$ by dropping the equality sign as we actually only need to consider the interior of the sets involved:

$$
2 A+A^{2}>\frac{1}{4\left(x_{k}-\delta\right)^{2}} \Leftrightarrow x_{k}>\delta+\frac{1}{2 \sqrt{A^{2}+2 A}} .
$$


C. The image of $\mathbf{H}_{-\delta}$ under $s_{k}^{(2)}\left(y_{1}, y_{2}\right)=\left(1+y_{1}\right) /\left(b_{k}+y_{2}\right)$.

For fixed $y_{1}$ we find the image as under $\mathbf{A}$, (75); multiplying by $1+y_{1}$ this gives the image as a disk with center and radius as below

$$
M=\frac{1+y_{1}}{2\left(x_{k}-\delta\right)}, R=\frac{\left|1+y_{1}\right|}{2\left(x_{k}-\delta\right)},
$$

and to find a value in $\mathbf{H}_{-\delta}$ again, we need

$$
\operatorname{Re} M-R>-\delta
$$

leading to

$$
2 \delta\left(x_{k}-\delta\right)>-\left(\operatorname{Re}(1+y+1)-\left|1+y_{1}\right|\right) .
$$

Using the bound following from $y_{1} \in \mathbf{P}$ this implies

$$
2 \delta\left(x_{k}-\delta\right)>A \Leftrightarrow x_{k}>\delta+\frac{A}{2 \delta} .
$$

Putting the conditions together, we have to find $\delta>0, A>0$ for which (80), (84) are satisfied, but give a lower bound (73) for the real value of $b_{k}$ that is as small as possible. The conditions

$$
x_{k}>\delta+\frac{1}{2 \sqrt{A^{2}+2 A}}, x_{k}>\delta+\frac{A}{2 \delta}
$$

can be 'optimized' in the following way.

The second inequality is, for fixed $A$, a function of $\delta>0$ having a global minimum at

$$
\delta=\sqrt{\frac{A}{2}},
$$

and it turns out that a lower bound for $x_{k}$ can be given by

$$
\max \left(2 \sqrt{\frac{A}{2}}, \sqrt{\frac{A}{2}}+\frac{1}{2 \sqrt{A^{2}+2 A}}\right) .
$$

Because $\sqrt{A / 2}$ is strictly increasing and $1 /\left(2 \sqrt{A^{2}+2 A}\right)$ strictly decreasing for $A>0$, the optimal choice is found for

$$
\sqrt{\frac{A}{2}}=\frac{1}{2 \sqrt{A^{2}+2 A}},
$$

i.e.

$$
2 A^{3}+4 A^{2}-1=0 .
$$

This cubic equation has a unique positive root and inserting the values found for $\delta$ and $A$, we arrive at (31), (32). 


\section{Concluding remarks}

Most of the calculations given above can be generalized to the case of $n$-fractions with $n \geq 3$. The extension of Lemma 2, however, seems to be more difficult and of course there is still the question whether the continued fraction in Theorem 7 does converge or not. This will be studied in a forthcoming paper. Furthermore, the author is indebted to the anonymous referee, whose remarks led to clarification of some obscure steps in the proofs.

\section{References}

1. Perron, O.: Grundlagen für eine Theorie des Jacobischen Kettenbruch-algorithmus. Math. Ann. 1-76 (1907)

2. Skorobogat'ko, V. Ya., Dronyuk, N.S., Bobik, O.I., Ptashnik, B.I.: Branched continued fractions and their applications. Dop. Akad. Nauk Ukr. RSR, Ser. A, No. 2, 131-133 (1967)

3. de Bruin, M.G.: Generalized C-fractions and a multidimensional Padé table. Dissertation, University of Amsterdam, Amsterdam (1974)

4. de Bruin, M.G.: Convergence along steplines in a generalized Padé table. In: Saff, E.B., Varga, R.S. (eds.) Padé and Rational Approximation, pp. 15-22. Academic Press, New York (1977)

5. Skorobogat'ko, V. Ya.: Theory of Branched Continued Fractions and its Applications in Computational Mathematics (in Russian). Nauka, Moscow (1983)

6. Wimp, J.: Computation with Recurrence Relations, Applicable Mathematics Series, Advanced Publishing Program. Pitman, London/Massachusetts (1984)

7. Levrie, P.: Pringsheim's theorem for generalized continued fractions. J. Comput. Appl. Math. 14, 439-445 (1986)

8. Bodnar, D.I.: Branched Continued Fractions (in Russian). Naukova Dumka, Kiev (1986)

9. Lorentzen, L., Waadeland, H.: Continued Fractions with Applications, Studies in Computational Mathematics, vol. 3. North-Holland, Amsterdam (1992)

10. Bodnar, D.I.: On the convergence of branched continued fractions. J. Math. Sci. 97(1), 3862-3871 (1999) [translated from Mathematychni Metody ta Fizyko-Mechanichni Polya. 4(1), 117-126 (1998)] 